\title{
FORTALEZAS Y DEBILIDADES DE LAS IMPLEMENTACION DE LAS TICS EN EL SISTEMA JUDICIAL DE CARTAGENA. ${ }^{1}$
}

Strengths and weaknesses of the implementation of the TICS in the judicial system of Cartagena

Ingrid Montes Alvarino ${ }^{2}$

Saray García Viaña ${ }^{3}$

Andrés Mauricio Cabarcas Argumedo ${ }^{4}$

\section{RESUMEN}

Hoy en día la globalización ha tenido un impacto y una acogida favorable en cuanto a las implementaciones de las TICS en los diferentes campos en los que se utiliza esta. El siglo XXI es el tiempo en el cual los seres humanos se han beneficiado estas herramientas tecnológicas, dichas herramientas han contribuido al mejoramiento de las relaciones y diferentes actividades a desarrollar día a día.

En este caso en concreto se puede observar como el beneficio para la implementación de las TICS en el sistema judicial en Cartagena, sirve de apoyo a los procedimientos, actuaciones en las que los usuarios pueden estar actualizados en cuanto a sus procesos, para así descongestionar los despachos judiciales.

Palabras claves: Globalización, sistema judicial, descongestión,TICS, tecnologías.

\section{ABSTRACT}

Nowadays the globalization has had an impact and a favorable reception as for the implementations of the TICS in the different fields in which this one is in use. The 21 st century is the time in which the human beings have benefited these technological tools; the above mentioned tools have helped to the improvement of the relations and different activities to develop day after day. In this case, in I make concrete it is

\footnotetext{
${ }^{1}$ Articulo resultado del Proyecto académico de Trabajo PAT Colectivo VI Semestre IIP-2018, del Programa de Derecho de la Corporación Universitaria Rafael Núñez, campus Cartagena.

${ }^{2}$ Docente del Programa de derecho de la Corporación Universitaria Rafael Núñez, campus Cartagena, orientadora del Seminario de Investigación como espacio de formación VI Semestre IIP-2018. E-mail: ingrid.montes@curnvirtual.edu.co

${ }^{3}$ Estudiante de VI Semestre Programa de Derecho de la Corporación Universitaria Rafael Núñez, campus Cartagena. E-mail: sgarciav13@curnvirtual.edu.co

${ }^{4}$ Estudiante de VI Semestre Programa de Derecho de la Corporación Universitaria Rafael Núñez, campus Cartagena. E-mail: acabarcasa13@curnvirtual.edu.co
} 
possible to observe as the benefit for the implementation of the TIC in the judicial system in Cartagena, uses as support to the procedures, actions in which the users can be updated as for his processes, this way to clear the judicial offices.

Keywords: Globalization, judicial system, decongestion, TICS, technologies.

\section{INTRODUCCIÓN}

Este artículo se hace referencia a las debilidades y fortalezas de la implementación de la TIC en el sistema judicial en la ciudad de Cartagena, comenzando por comprender que las TIC se han convertido en una figura muy significativa e indispensable para la sociedad, ya que estas han sido de gran contribución para la evolución y desarrollo de la misma, las TIC son tecnologías que permiten acceder, producir, guardar, presentar y transferir información y aparte de eso nos bridan una solución eficiente y eficaz en los problemas del diario vivir. Este artículo se hizo con el interés de comprender en que consiste la implementación de las TIC en el sistema judicial de Colombia, brindándonos una serie de conocimientos recolectados de varias fuentes.

Se mostrará dentro del documento cuales leyes reglamentan, definen, implementan y promulgan, las tecnologías de la información y la comunicación, poniendo al conocimiento de todas las personas de quien sea interés, como se determinaría de manera general las políticas públicas que regirían este sector, y de la misma forma cómo se daría el uso eficiente de las redes dentro de los despachos judiciales y demás entidades, la efectiva planeación y gestión para facilitar un acceso eficaz y eficiente a los habitantes del territorio nacional.

\section{MATERIALES Y MÉTODOS}

Nuestro método de investigación es deductivo debido a que parte de una o más explicaciones para llegar a una conclusión, su aplicación es cualificada debido que tomamos las investigaciones de los libros, artículos, documentos que se encuentran en las fuentes bibliográficas y procedimos a nuestra interpretación sobre lo investigado.

Las técnicas e instrumentos de recolección, serán los libros, documentos y artículos que encontramos en las fuentes bibliográficas.

\section{IMPACTO DE LAS TIC EN EL SISTEMA JUDICIAL DE CARTAGENA}

De acuerdo a Montes (2017):

El uso de las Tecnologías de la Información y el Conocimiento, en adelante TIC, tiende a incidir en todas las esferas de la sociedad y el impacto benéfico de las mismas ha sido de gran auge e importancia, al configurarse también como mecanismo de inclusión social y de lucha contra la pobreza. (p. 171) 
(...) En Colombia a partir de la ley 1341 de 2009 o Ley TIC, se consagra la protección de los derechos de los usuarios de las TIC, como principio orientador de las Tecnologías de la información y las comunicaciones, las cuales son una política de Estado "que involucra a todos los sectores y niveles de la administración pública y de la sociedad, para contribuir al desarrollo educativo, cultural, económico, social y político e incrementar la productividad, la competitividad, el respeto a los Derechos Humanos inherentes y la inclusión social. (Montes, 2017, pág. 171)

Como respuesta a nuestro primer objetivo, tenemos que este sistema se implementó o se incorporó en el uso de las tecnologías como ayuda para cumplir eficientemente con sus objetivos, ya sea en soluciones para el mejoramiento de la justicia, en la ciudad de Cartagena generando una fortaleza a los despachos judiciales y al litigio estas herramientas electrónicas informan y comunican de manera rápida y ágil los procesos que se llevan a cabo permitiendo una interconexión en el traspaso de información y manejo adecuado de la misma. La implementación de estas, analizándolo desde la gestión judicial aportando un buen servicio de justicia con respuestas prontas que prioricen las necesidades de los usuarios en aras de consolidar lo que el Estado pretende establecer, partiendo de esta consigna obtuvimos una respuesta distinta a lo esperado con las TICS en nuestra ciudad.

En relación con el ahorro de tiempo, estas herramientas cumplen unos aspectos fundamentales y muy importantes, debido a que por medio de estas se evita la asistencia continua a los despachos judiciales brindando la posibilidad a las partes de tener un acercamiento directo con los procesos a través de la sistematización del mismo. ${ }^{5}$ Otro papel importante que cumplen las Tics en el sector justicia, es precisamente que estas minimizan el uso del papel, ya que se permiten presentar recursos por medios electrónicos y esto contribuye a la descongestión del despacho en materia de espacio y también a la tarea de la administración actual de conservar el medio ambiente. Así mismo, en relación a la trasparencia su papel es muy relevante, debido a que la utilización de estas garantiza al usuario que su proceso se resuelve de manera ágil, segura y confiable.

Las TICS en las organizaciones abre nuevas oportunidades para el mejoramiento e interactividad entre diferentes entidades, asegurando el manejo más eficiente de los recursos. El uso de las TICS puede contribuir al crecimiento de la productividad del trabajo por medio de la intensificación del capital. La utilización adecuada de las TICS en una organización requiere cambios en la manera como se desarrollan las labores, creando nuevas composiciones de la mano de obra, perfiles más especializados y competencias orientadas al uso de medios tecnológicos para aumentar la eficiencia en los resultados institucionales y mejorar la oportunidad, la eficacia, la transparencia y la confiabilidad de la prestación de los servicios de administración de justicia en el país; prestar una

${ }^{5}$ Sánchez Upegui, A. A. (agosto, 2009). Nuevos modos de interacción educativa: análisis lingüístico de un foro virtual. Revista Educación y Educadores, 2(2), 29-46. Recuperado de http://educacionyeducadores.unisabana.edu.co 
pronta, ágil y oportuna justicia; mejorar la calidad de las decisiones judiciales; aumentar la productividad de los despachos judiciales y hacer una gestión eficiente de los procesos administrativos judiciales.

Como respuesta a nuestro segundo objetivo, encontramos que los beneficios que trae el ministerio de comunicación tiene la finalidad de atender las necesidades resultantes de las consecuencias de los cambios por la tecnología aplicadas a las telecomunicaciones y conformar el respectivo sector de la rama ejecutiva del poder público. Las cuales han sido muy favorable para los litigantes ya que le permite de cualquier modo acceder a la información eficaz y rápida, este sistema se implementó o se instaló con el objetivo descongestión el aparato judicial, dando un buen Manero en los papeles archivos incluso en los procesos que se están realizando los litigantes dentro de la administración de justicia regulando los trámites judiciales logrando un buen servicio en los despachos, por medio de eso los litigantes pueden guardar cualquier información sin necesidad que se vaya a perder como grabaciones, anexos, pruebas e incluso le permiten la utilización de los correos electrónico para comunicarle los procesos.

Estas herramientas son muy útil para ellos e incluso les cede cualquier ubicación fácil y más ágil de los distintos procesos organizados virtualmente con el determinado tramite o etapas así llevar a cabalidad la administración, vemos también las plataformas habilitadas para que verifiquen los estados y el avance de sus proceso, notificaciones reproductores de audio y video se hacen por medio electrónico, con esto se instaura una interconexión entre el sistema de justicia y el usuario de manera pronta lo que beneficia para el descongestionamiento masivo en los juzgados de la ciudad. Teniendo en cuenta que son múltiples los usos y servicios de las TICS que cumplen el papel de agilizar, dinamizar y simplificar los procesos judiciales mejorar los flujos de trabajo de los litigantes, mejorar las coordinación y comunicación entre servidores judiciales facilitar la supervisión y gestión de los procesos judiciales y reforzar los mecanismos de seguridad y de acceso a la información continua especifica resolver los problemas de falta de recursos físicos, humanos y tecnológicos la creciente demanda mejorar la interoperabilidad del sistema, consiguiendo que los órganos judiciales puedan intercambiar información, de manera ágil, segura y confiable y pasar de un mundo físico a un mundo digital aportar en la consolidación del expediente digital y reducción del uso del papel.

Por último, nuestro tercer objetivo da respuesta a las fortalezas que se dan a las TICS. Se trata sobre la utilización de avanzada tecnología al servicio de la administración de justicia donde pueden utilizar medios electrónicos, informáticos, telemáticos. Respecto a ello se encontró el uso de las cámaras de video, celulares, computadores, micrófono, video vin, anexando a ello el correo electrónico, scanner, los litigantes que se han tomado un desarrollo vital este sistema, 
el programa como Microsoft Word, Excel; en este orden todo ellos permiten ya sea la relación, conexión o el intercambio de mensajes, el registro de audiencia en audio y video. Estos medios facilitan la comunicación y la información como a los funcionarios que tenga una conexidad en los procesos que llevan los litigantes, como la interacción de las informaciones que se hagan de una manera rápida y fácil, en la rama judicial de Cartagena se utilizan de la misma forma el celular, computadores, cámaras de video, micrófonos, video vin, scanner. Como diferentes programas, correo electrónico, video conferencias vía Skype, instalaciones de nodos e impresoras. Ejemplo de su aprovechamiento es la implementación del registro de procesos para que los litigantes puedan estar informados, en algunos despachos como juzgados sean administrativo penal civil en todas las áreas le permiten fortalecer las gestiones a la laboral de los abogados, lo que más impacta es que ha sido de grandes beneficios, por medio de la existencia de las notificaciones o los estados electrónicos, también notificaciones por estados que se hacen atreves de la página web de la rama judicial.

Este programa cuenta con una correspondencia llamado sigobius o sistema de Gestión de Correspondencia y Archivo de Documentos Oficiales, en el cual toda correspondencia que se recibe es incluida en este, pero cuando van a ser respondidas se hacen en esta resolución, oficio etc. Y se comunica por correo electrónico, salvo cuando la ley dice que debe ser notificado personalmente.

Que nos garantiza una efectividad en cada proceso que estamos desarrollando de manera rápida y efectiva por medio de estas herramientas, que reúne todos los beneficios para mejorar el área de trabajo de los litigantes, incluso ha hecho un impacto en cualquier área laboral ya que permite una acceso rápido y directo a las necesidades, como de guarda en los archivos las demandas pruebas que son necesaria en una audiencia y que en el momento se nos presenta cualquier circunstancia, y hay tenemos todo en las base de datos guardo para agilizar el proceso.

\section{CONCLUSIONES}

El revolucionario impacto de las TICS en la sociedad determina un cambio estructural pues ha transformado la forma como se produce, divulga y utiliza la información, permitiendo la movilidad y rapidez de los procesos, disminuyendo los costos de transacción y favoreciendo la descentralización.

Los grados de implementación en el uso de las TIC son el tratamiento de la información, el tratamiento de expedientes judiciales, la interacción entre usuarios y servidores judiciales y el juicio digital para la toma de decisiones. La Administración de Justicia electrónica permitirá prestar un mayor número de servicios, mejorar la calidad, racionalizar esfuerzos y recursos, así como abrir nuevos canales de actuación, comunicación e información accesibles a todos. 
Lo anterior apunta al fortalecimiento institucional de la Rama Judicial en el trabajo de los litigantes, que tiene como propósito afianzar y posicionar a la rama judicial como una organización estable, con medios suficientes y adecuados para garantizar la prestación del servicio público de justicia en términos de oportunidad, eficiencia, eficacia y calidad, de tal forma que responda a las exigencias de la sociedad.

Finalmente, y en relación con las debilidades encontradas dentro de la administración de justicia, se determina que lamentablemente muchos funcionarios y empleados judiciales no saben manejar las TICS y generalmente en los casos en que el servicio de luz eléctrica falla, de inmediato se detiene el sistema judicial, de ahí que se debe trabajar para garantizar que la prestación de estos servicios sean eficaces teniendo en cuenta que se debe contar con un grupo de profesionales idóneos y capaces que manejen estas herramientas.

\section{REFERENCIAS BIBLIOGRAFICAS}

AZ Revista de Educación y Cultura (14 de abril de 2014). Abogados y estudiantes de Derecho frente a las TIC. México: Zenago Editores. Recuperado de https://educacionyculturaaz.com/ abogados-y-estudiantes-de-derecho-frente-a-las-tic/

Carbonell, M. (2009) Cartas a un estudiante de Derecho. Madrid, España: Taurus Ed.

Fernández, F. (20017). La gestión de la nueva comunicación interna. Análisis de la aplicación de las tecnologías de la información en los procesos de comunicación interna de las universidades de la Comunidad Valenciana. Tesis Doctoral presentada en la Universitat Jaume I. Departament de Filosofia, Sociologia i Comunicació Audiovisual i Publicitat. Tutor: López, R. Recuperado de https://www.tdx.cat/handle/10803/10464\#page=1

Gaviria, S. (2016) Se requiere de una superintendencia TIC que vigile y controle este sector. Colombia, Periódico El Universal: Tecnología, 2 de mayo de 2016. Recuperado de https:// www.eluniversal.com.co/tecnologia/se-requiere-de-una-superintendencia-tic-que-vigiley-controle-este-sector-225157-CQEU330615

Informe al Congreso de la República de Colombia 2011 (2012) Capítulo 5: Tecnologías de la Información y las Comunicaciones (TIC) para la justicia: 73-85. Recuperado de https://www. ramajudicial.gov.co/documents/10228/1468180/2011+CAP\%C3\%8DTULO+5-TECNO $\mathrm{LOG} \% \mathrm{C} 3 \% 8 \mathrm{DAS}+\mathrm{DE}+\mathrm{LA}+\mathrm{INFORMACI} \% \mathrm{C} 3 \% 93 \mathrm{~N}+\mathrm{Y}+\mathrm{LAS}+\mathrm{COMUNICACIONES}$. pdf/42e108a8-6fa3-4967-8911-70e4b5d5d3e8 
Lillo, R. (2009) Indicadores de CEJA: El rol de las TIC en una justicia para En Sistemas Judiciales. Publicación semestral del Centro de Estudios de Justicia de las Américas, CEJAAño 9, Número 16. Chile: CEJA: 6-18

Lillo, R. (2010) El Uso de Nuevas Tecnologías en el Sistema Judicial: experiencias y precauciones. En: José A. Caballero, Carlos Gregorio y Linn Hammergren (comp.), "Buenas Prácticas para la Implementación de Soluciones Tecnológicas en la Administración de Justicia”, IIJusticia: 121-144.

Londoño, N. (2010) El uso de las TIC en el proceso judicial: una propuesta de justicia en línea. En Revista Facultad de Derecho y Ciencias Políticas, 40 (112): 123-142

Montes Alvarino, I. (2017), Defensas del usuario de comunicaciones en Colombia:un camino tortuoso en la era digital. Revista Jurídica Mario Alario D'Filippo, 9, (17):169184. Recuperado de DOI: https://doi.org/10.32997/2256-2796-vol.9-num.17-2017-1547

Pallares, Y (2012) Como surgieron las TICS. En: Gestión de TIC. Recuperado de http:// planeacionygestiondetics.blogspot.com/2012/11/como-surgieron-las-tic.html

Rodríguez, M. (2015) Las TICs en Colombia. Repositorio del Colegio de Estudios Superiores de Administración/CESA. Bogotá, Colombia. Recuperado de https://repository.cesa.edu.co/ bitstream/handle/10726/797/1044.pdf?sequence=2\&isAllowed=y 